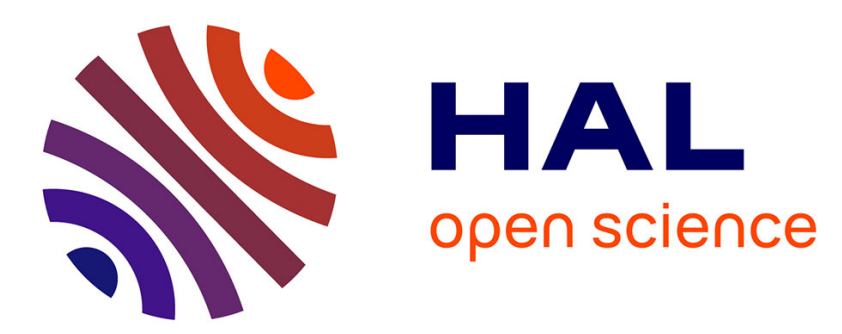

\title{
Investigation of the interwire energy transfer of elastic guided waves inside prestressed cables
}

\author{
Fabien Treyssede
}

\section{To cite this version:}

Fabien Treyssede. Investigation of the interwire energy transfer of elastic guided waves inside prestressed cables. Journal of the Acoustical Society of America, 2016, 140 (1), 13p. 10.1121/1.4955080 . hal-01589912

\section{HAL Id: hal-01589912 \\ https://hal.science/hal-01589912}

Submitted on 19 Sep 2017

HAL is a multi-disciplinary open access archive for the deposit and dissemination of scientific research documents, whether they are published or not. The documents may come from teaching and research institutions in France or abroad, or from public or private research centers.
L'archive ouverte pluridisciplinaire HAL, est destinée au dépôt et à la diffusion de documents scientifiques de niveau recherche, publiés ou non, émanant des établissements d'enseignement et de recherche français ou étrangers, des laboratoires publics ou privés. 


\title{
Investigation of the interwire energy transfer of elastic guided waves inside prestressed cables
}

\author{
Fabien Treyssède ${ }^{1, a)}$ \\ LUNAM Université, IFSTTAR, GERS, GeoEND, F-44340 Bouguenais, France
}

(Dated: 6 January 2016)

\begin{abstract}
Elastic guided waves are of interest for the non-destructive evaluation of cables. Cables are most often multiwire structures, and understanding wave propagation requires numerical models accounting for the helical geometry of individual wires, the interwire contact mechanisms and the effects of prestress. In this paper, a modal approach based on a so-called semi-analytical finite element method and taking advantage of a biorthogonality relation is proposed in order to calculate the forced response under excitation of a cable, multi-wired, twisted and prestressed. The main goal of this paper is to investigate how the energy transfers from a given wire, directly excited, to the other wires in order to identify some localization of energy inside the active wire as the waves propagate along the waveguide. The power flow of the excited field is theoretically derived and an energy transfer parameter is proposed to evaluate the level of energy localization inside a given wire. Numerical results obtained for different polarizations of excitation, central and peripheral, highlight how the energy may localize, spread or strongly change in the cross-section as waves travel along the axis. In particular, a compressional mode localized inside the central wire is found, with little dispersion and significant excitability.
\end{abstract}

\section{INTRODUCTION}

Multi-wire cables are widely used in engineering applications. For instance, they are used in civil engineering for prestressing and post-tensioning concrete, or as load carrying members for cable bridges. In electrical engineering, multi-wire cables are used in power transmission lines.

Such structures are subjected to environmental degradations (corrosion, temperature, wind). Maintaining their safety becomes essential. In order to assess their structural health, the development of non destructive evaluation (NDE) techniques is necessary. Guided wave based methods are of particular interest because these waves can propagate over long distances and enhance the inspection range with a single measurement.

In practice, the analysis of elastic guided waves requires modeling tools due to their dispersive and multimodal nature. Experiments have shown that a multiwire cable cannot generally be modeled as an effective equivalent cylinder ${ }^{1-3}$. With multi-wire cables, the modeling task must indeed face several difficulties, among which the helical geometry of individual wires, the contact between wires and the presence of huge tensile loads. Some recent progress have been made by subsequently accounting for these difficulties in numerical models of wave propagation $^{4-8}$. These numerical models are based on a semi-analytical finite element (SAFE) method, which has been widely used for the study of straight waveguides of arbitrary shape $\mathrm{e}^{9-12}$. For the modeling of helical multi-wire strands, the SAFE formulation must be written in twisting coordinates and account for prestress. Numerical results have been shown to compare well with

\footnotetext{
a) fabien.treyssede@ifsttar.fr
}

experimental results obtained for longitudinal modes in seven-wire strands, in particular as far as the so-called 'notch frequency' phenomenon ${ }^{1,2}$ is concerned (such a phenomenon, related to a missing frequency band in experiments, indeed corresponds to a curve veering and is typical of strands ${ }^{6}$ ).

However, some modeling works are still required in order to improve the understanding of wave propagation inside cables. As far as it goes, the effort has mainly focused on the free response of cables regardless excitation. The forced response of cables under excitation has been barely considered ${ }^{13}$. Some transient three-dimensional finite element models have been proposed ${ }^{14,15}$, but these models are costly from a computational point view, which limits the analysis and the physical interpretation of results.

Another specificity of multi-wire cables is that they can naturally be considered as substructured waveguides (wires being substructures). Of particular interest in this paper is the transfer of energy that occurs between substructures. More precisely, the goal of this paper is to theoretically investigate how the energy transfers from a given wire, directly excited, to the other wires in order to identify some localization of energy, if any, inside a single wire as the waves propagate along the waveguide. To this end, the theory presented in Ref. 8 is extended to account for acoustic sources in the SAFE formulation, which only requires the discretization of the two-dimensional crosssection of the waveguide and enables an efficient computation of the forced response based on a modal approach.

In this paper, we will focus our attention on the socalled seven-wire strand, constituted by one central cylindrical wire surrounded by six helical peripheral ones (see Fig. 1). Such a structure is widely used in modern bridges. It should be noticed that the method developed in this paper can be applied to any multi-wire helical structures composed of several layers of helical wires 


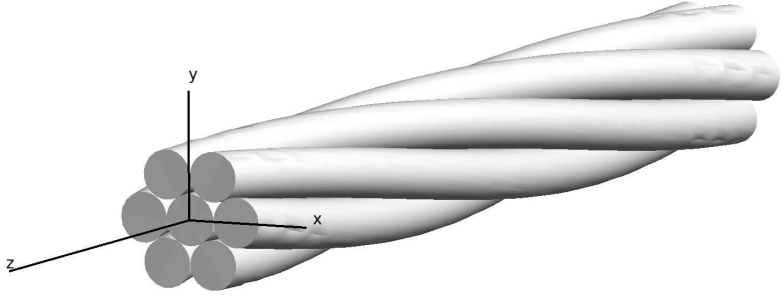

FIG. 1: Geometry of a seven-wire strand.

wrapped with the same twisting rate around a straight axis. Yet, the method is not applicable to the case of crosslay strands (involving both positive and negative torsion) or double helical structures (composed of one central strand wrapped by several helical peripheral strands $)^{7}$.

Section 2 briefly reviews the main theoretical results for implementing the SAFE method with twisting coordinates, prestress and contact width effects. In Section 3 , the forced response of twisted prestressed waveguides is calculated thanks to a modal approach by taking advantage of a biorthogonality relation. The power flow of the excited field is then theoretically investigated and an energy transfer parameter is proposed in order to evaluate the level of energy localization inside a specific wire. Section 4 finally gives numerical results obtained for several kinds of excitation, applied to the central wire or to a peripheral wire, and highlights how the energy may localize, spread or change in the cross-section as waves travel along the axis far from the source.

\section{THEORETICAL BACKGROUND}

\section{A. Dynamics of prestressed structures}

The analysis of the dynamics of prestressed structures requires a non linear theory. It can be decomposed into a static problem, solved independently, and a linearized dynamic problem superimposed on this prestressed state. Three configurations must be distinguished: the initial configuration (without initial stress), the static prestress configuration and the final configuration (including dynamics). Subscripts $i$ and 0 will be used to distinguish variables in the initial and in the prestress configurations respectively.

In this paper, one will assume small strain both in statics and dynamics so that material non linearities are neglected and the material remains linearly elastic. The account for prestress on dynamics yet requires geometrical non linearities of the predeformed configuration.

One assumes small displacements superimposed onto the prestressed static state, with a time harmonic $e^{-i \omega t}$ dependence. Expressing variables in the prestress configuration, the equilibrium equations of elastodynamics un- der prestress are given in the frequency domain by ${ }^{16,17}$ :

$$
\nabla_{0} \cdot\left(\sigma+\nabla_{0} \mathbf{u} \cdot \sigma_{0}\right)+\rho_{0} \omega^{2} \mathbf{u}=\mathbf{f}_{v}
$$

with the natural boundary condition:

$$
\left(\sigma+\nabla_{0} \mathbf{u} \cdot \sigma_{0}\right) \cdot \mathbf{n}_{0}=\mathbf{f}_{s} \text { on } \partial V_{0}
$$

where $\mathbf{u}, \sigma=C_{0}: \epsilon$ and $\epsilon=\left(\nabla_{0} \mathbf{u}+\nabla_{0} \mathbf{u}^{T}\right) / 2$ denote the small dynamic perturbations of the displacement vector, stress tensor and strain tensor respectively. $C_{0}$ and $\rho_{0}$ denote the elasticity tensor and the material density. $\sigma_{0}$ is the Cauchy prestress, i.e. the stress tensor associated with the prestressed state. $\nabla_{0}$ refers to the gradient operator with respect to the prestressed configuration. $\mathbf{f}_{v}$ represents acoustic sources inside the prestressed structural volume $V_{0} . \mathbf{f}_{s}$ is the traction vector applied on the boundary $\partial V_{0}$ of the structure of unit outward normal $\mathbf{n}_{0}$.

\section{B. Semi-analytical finite element method}

The analysis of guided waves inside helical strands requires a specific curvilinear coordinate system, called twisting coordinate system. Such a system has constant non zero torsion but zero curvature, and hence corresponds to a particular case of helical system. In Ref. 6 , it has been shown that a twisting system allows to preserve translation invariance in seven-wire strands, which hereby yields a theoretical proof for the existence of guided waves in such structures. With this kind of system, the cross-section plane remains perpendicular to the straight axis but rotates around this axis by following peripheral wires. Provided that the central wire is circular and isotropic, the cross-section of the whole structure and its material properties remain translationally invariant in a twisting system. The torsion of the twisting system is given by $\tau_{0}=2 \pi / L_{0}$, with $L_{0}$ denoting the helix pitch of prestressed peripheral wires. This section brielfy reviews the main equations of the SAFE method written in a twisting system and including prestress effects. Further details can be found in Refs. 7 and 8 .

Let us denote $z$ the straight axis of the waveguide, fixed to the Cartesian system, $(x, y)$ the cross-section twisting coordinates, $k$ the axial wavenumber. The application of a SAFE method consists in assuming an $e^{i k z}$ dependence of acoustic fields before finite element (FE) discretization. Therefore, only the two-dimensional cross-section in the $(x, y)$ plane of the structure has to be meshed. One points out that the $e^{i k z}$ field dependence implies that axial variables must be separable from transverse variables in the governing equations of motion (this separation of variable is actually possible thanks to the proof of translational invariance along the $z$-axis that holds in a twisting system ${ }^{6,18}$ ).

The application of the SAFE method can be summarized as follows. First a spatial Fourier Transform is 
applied to Eqs. (1) and (2), here written in the twisting system. Then, the weak form is derived by integrating the equations on the cross-section, denoted as $S_{0}$. The boundary of $S_{0}$ is denoted as $\partial S_{0}$. Finally, the cross-section is discretized by standard $2 \mathrm{D}$ FE. The SAFE method finally leads to the following matrix system governing wave propagation inside prestressed helical strands:

$$
\left\{\mathbf{K}_{1 \sigma}-\omega^{2} \mathbf{M}+i k\left(\mathbf{K}_{2 \sigma}-\mathbf{K}_{2 \sigma}^{T}\right)+k^{2} \mathbf{K}_{3 \sigma}\right\} \tilde{\mathbf{U}}=\tilde{\mathbf{F}}_{v}+\tilde{\mathbf{F}}_{s} .
$$

For paper self-containedness, the expressions of element matrices involved in the left hand side are recalled in Appendix A. The element vectors involved in the right hand, corresponding to acoustic sources, are given by:

$$
\tilde{\mathbf{F}}_{v}^{e}=\int_{S_{0}^{e}} \mathbf{N}^{e T} \tilde{\mathbf{f}}_{v} d x d y, \quad \tilde{\mathbf{F}}_{s}^{e}=\int_{\partial S_{0}^{e}} \mathbf{N}^{e T} \tilde{\mathbf{f}}_{s} d s
$$

The tilde notation is used to denote spatial Fourier transform of acoustic fields, with the following convention: $\tilde{f}(k)=\int_{-\infty}^{+\infty} f(z) e^{-i k z} d z$. For clarity, the finite element approximation of the displacement vector $\tilde{\mathbf{u}}$ is given on one two-dimensional element $e$ by: $\tilde{\mathbf{u}}(x, y)=\mathbf{N}^{e}(x, y) \tilde{\mathbf{U}}^{e}$, where $\mathbf{N}^{e}$ is the matrix of the shape functions and $\tilde{\mathbf{U}}^{e}$ is the vector of nodal displacements, with 3 degrees of freedom per node, written in the orthonormal Serret-Frenet basis associated with the twisting system.

Equations (3), (4) and (A1) can be viewed as the socalled linearized updated Lagrangian formulation of nonlinear mechanics (see e.g. Ref. 19), here extended to twisting coordinates and adapted to a SAFE formulation.

\section{Static prestress state and contact modeling}

The first step of the analysis consists in computing the static prestress state of the seven-wire strand loaded by a constant tensile strain. The rotational strain of the strand is set to zero. One assumes that the influence of static non-linearities can be neglected on dynamics and the static prestress state will hence remain linear. Its computation can be achieved efficiently using a homogenization method specifically written in twisting coordinates $^{7}$. This allows to exploit the translational invariance property, and hereby, to restrict the problem to the cross-section.

It can be shown that the prestressed state subjected to a prescribed axial strain, denoted as $\epsilon$, can be determined by a linear static computation: $\mathbf{K}_{0} \mathbf{U}_{0}=\mathbf{F}_{0}$, where $\mathbf{U}_{0}$ is the static nodal displacement vector, $\mathbf{F}_{0}$ is the external load vector corresponding to an applied axial strain and the expressions of $\mathbf{K}_{0}^{e}$ and $\mathbf{F}_{0}^{e}$ are recalled in Appendix A. More details can be found in Ref. 7 .

Then, the Cauchy prestress $\sigma_{0}$ necessary for the calculation of SAFE matrices can be post-processed from the solution of the linear static computation.

However, it must be emphasized that the helical geometry of peripheral wires yields a radial compression of the central wire when a tension is applied in the axial direction. As a consequence, the interwire contact width tends to increase as the tensile load increases. This phenomenon is neglected when the linear static problem $\mathbf{K}_{0} \mathbf{U}_{0}=\mathbf{F}_{0}$ is solved in a unique step. Instead, an iterative procedure must be used in order to account for the mechanics of contact, which is inherently non linear.

The contact modeling approach used in this paper is based on a node-to-node contact procedure together with a direct elimination method ${ }^{20}$. Matching meshes are used inside each interfacial zones where the central wire and each helical wire can possibly come into contact. Note that no contact occurs between peripheral wires ${ }^{7,8}$. For the $2 \mathrm{D}$ cross-section FE model used in this paper, the computation starts with single point contacts between wires. Then as the axial load is incremented, contact pairs of nodes are successively formed: once the gap between a pair of nodes is closed, the continuity of displacement is enforced at these nodes by the direct elimination method. Stick contact conditions are assumed (infinite friction), so that the displacement continuity at contact nodes is enforced along the three directions.

The iterative procedure can be summarized by the following linear incremental equation for each incremental load step $j$ :

$$
\mathbf{K}_{0}^{j} \Delta \mathbf{U}=\frac{\Delta \epsilon}{\epsilon} \mathbf{P}^{j \mathrm{~T}} \mathbf{F}_{0}, \quad \mathbf{K}_{0}^{j}=\mathbf{P}^{j \mathrm{~T}} \mathbf{K}_{0} \mathbf{P}^{j}
$$

where $\mathbf{P}^{j}$ denotes the projection matrix which reduces the displacement degrees of freedom that are in contact at step $j, \Delta \mathbf{U}$ and $\Delta \epsilon$ are the incremental displacement and incremental axial strain respectively. The static displacement $\mathbf{U}_{0}$ for a prescribed strain $\epsilon$ is finally obtained by the sum of the incremental displacement.

\section{INTERWIRE ENERGY TRANSFER OF THE FORCED RESPONSE}

\section{A. Free response}

The free response problem is considered in order to compute the propagation modes. Setting $k$ to a fixed value, the homogeneous SAFE system (3) is a linear eigenproblem for finding the eigenvalue $\omega$. However since the forced response will be given as a function of $z$ and $\omega$ (see next subsection), the eigenproblem (3) must necessarily be solved for each frequency instead. Setting the frequency $\omega$ to a fixed value, Eq. (3) is a quadratic eigenproblem for the eigenvalue $k$.

For an eigenvalue $k,-k$ is also an eigenvalue. This property results from the symmetry of $\mathbf{K}_{1 \sigma}, \mathbf{K}_{3 \sigma}$ and $\mathbf{M}$. Hence, the eigenproblem has then two kinds of eigensolutions: $\left(k_{m}, \mathbf{U}_{m}^{+}\right)$and $\left(-k_{m}, \mathbf{U}_{m}^{-}\right), m=1, \ldots, M$, representing $M$ modes traveling in the positive direction and $M$ modes in the negative direction.

Quadratic eigenproblems are more difficult to handle with standard numerical eigensolvers. A rather 
well-known procedure consists in recasting the quadratic eigenproblem as a linear one $\mathrm{e}^{21}$. In this paper, the following linear form is adopted:

$$
(\mathbf{A}-k \mathbf{B}) \hat{\mathbf{U}}=\hat{\mathbf{F}}
$$

with:

$$
\begin{gathered}
\mathbf{A}=\left[\begin{array}{c}
\mathbf{0} \\
\mathbf{I} \\
-\left(\mathbf{K}_{1 \sigma}-\omega^{2} \mathbf{M}\right)-i\left(\mathbf{K}_{2 \sigma}-\mathbf{K}_{2 \sigma}^{T}\right)
\end{array}\right], \\
\mathbf{B}=\left[\begin{array}{cc}
\mathbf{I} & \mathbf{0} \\
\mathbf{0} & \mathbf{K}_{3 \sigma}
\end{array}\right], \hat{\mathbf{U}}=\left\{\begin{array}{c}
\tilde{\mathbf{U}} \\
k \tilde{\mathbf{U}}
\end{array}\right\}, \hat{\mathbf{F}}=\left\{\begin{array}{c}
\mathbf{0} \\
-\tilde{\mathbf{F}}
\end{array}\right\}
\end{gathered}
$$

Assuming a lossless material (i.e. real coefficients in $\mathbf{C}_{0}$ ), an interesting property with the linear form (6) is that the matrix $\mathbf{B}$ is Hermitian, which can be used to improve the numerical treatment by eigensolvers ${ }^{22}$.

In this paper, the dispersion curves will be plotted for the energy velocity as a function of frequency. The energy velocity of the $m$ th mode can be post-processed from SAFE matrices as follows ${ }^{8}$ :

$$
v_{e_{m}}=\frac{2 \omega \operatorname{Im}\left\{\mathbf{U}_{m}^{*}\left(\mathbf{K}_{2 \sigma}^{T}+i k_{m} \mathbf{K}_{3 \sigma}\right) \mathbf{U}_{m}\right\}}{\operatorname{Re}\left\{\mathbf{U}_{m}^{*}\left(\mathbf{K}_{1 \sigma}+\omega^{2} \mathbf{M}+i k_{m}\left(\mathbf{K}_{2 \sigma}-\mathbf{K}_{2 \sigma}^{T}\right)+k_{m}^{2} \mathbf{K}_{3 \sigma}\right) \mathbf{U}_{m}\right\}}
$$

where the superscripts $T$ and $*$ are used for the matrix transpose and the matrix complex conjuguate transpose respectively.

\section{B. Forced response}

The solution $\tilde{\mathbf{U}}=\tilde{\mathbf{U}}(k)$ of the problem under excitation is now expanded as a sum of the guided modes computed from the free response. As detailed in Ref. 23 for straight and unprestressed waveguides, the modal coefficients can be calculated by taking advantage of biorthogonality relations and applying the Cauchy residue theorem to obtain the solution in the $z$ domain. The SAFE biorthogonality relations are actually the discretized version of Auld's biorthogonality relations ${ }^{24}$.

Here despite the twist of the geometry and the presence of prestress, it must be emphasized that the SAFE matrices $\mathbf{K}_{1 \sigma}, \mathbf{K}_{3 \sigma}$ and $\mathbf{M}$ remain symmetric as in the standard case of straight and unprestressed waveguide problems (note that the additional terms due to the geometric stiffness obey such symmetry). On the other hand, the symmetry of these matrices is the only requirement for the results of Ref. 23. This amounts to say that Auld's biorthogonality relations can be extended to twisted and prestressed waveguides.

Therefore from Ref. 23, it can be shown that the solution as a function of $z>0$ can be expanded as follows:

$$
\mathbf{U}(z)=\sum_{m=1}^{M} \alpha_{m} \frac{\mathbf{U}_{m}}{\sqrt{P_{m m}}} e^{i k_{m} z}
$$

with:

$$
\alpha_{m}=\frac{i \omega}{4 \sqrt{P_{m m}}} \mathbf{U}_{m}^{*} \tilde{\mathbf{F}}\left(k_{m}\right)
$$

where the summation in Eq. (9) is performed over positive-going modes and $P_{m m}$ denotes the power flow of the $m$ th mode, which will be explicitly defined in the next subsection. The above solution is restricted to propagating modes $(k \in \mathbb{R})$ and neglects the contribution of non-propagating modes as well as any viscoelastic effect $\left(\mathbf{C}_{0}\right.$ must be real). Thus, $M$ should be understood as the number of propagating modes excluding those nonpropagating.

In this paper, attention is restricted to the far field. Given that the power flow of a non-propagating mode is equal to zero, the near-field region, where nonpropagating modes may have non-negligible contribution, is indeed of less interest for the present study.

As a side remark, it should be mentioned that twisting coordinates strongly modify the operators (see Eq. (A2)) and induce anisotropy in the equilibrium equations. As shown previously, Auld type relations are still applicable in this case. Yet other orthogonality relations of the literature may be not applicable with twist. For instance, Fraser type biorthogonality relations ${ }^{25,26}$ do not seem to exist in case of general anisotropic materials. Up to the author's knowledge, Fraser's relation has only been extended to particular anisotropy having at least one plane of elastic symmetry ${ }^{22,27}$.

\section{Power flow of the excited field}

Let us consider a subregion $\Gamma$ of the waveguide crosssection $S_{0}\left(\Gamma \subset S_{0}\right)$. The time averaged power flow of the acoustic field through the surface $\Gamma$ is defined by ${ }^{28}$ :

$$
\Pi_{\Gamma}=-\frac{i \omega}{4} \int_{\Gamma}\left(\mathbf{u}^{*} \mathbf{t}-\mathbf{t}^{*} \mathbf{u}\right) d x d y
$$


where $\mathbf{t}$ is the traction vector acting on $\Gamma$. In the present work, the expression of $\mathbf{t}$ must account for prestress and is given as follows ${ }^{8}: \mathbf{t}=\left(\sigma+\nabla_{0} \mathbf{u} \cdot \sigma_{0}\right) \cdot \mathbf{e}_{z}$.

After FE discretization, the above formula takes the following form:

$$
\Pi_{\Gamma}=-\frac{i \omega}{4}\left(\mathbf{U}_{\Gamma}^{*} \mathbf{T}_{\Gamma}-\mathbf{T}_{\Gamma}^{*} \mathbf{U}_{\Gamma}\right)
$$

where the subscripts $\Gamma$ on FE vectors denote their restriction to the degrees of freedom belonging to the subsurface $\Gamma$.

From the modal expansion (9), the power flow of the response generally depends on the propagation distance $z$ and can be rewritten as:

$$
\Pi_{\Gamma}(z)=\mathbf{q}(z)^{*} \mathbf{P}_{\Gamma} \mathbf{q}(z)
$$

where the modal components of $\mathbf{q}(z)$ and of the matrix
$\mathbf{P}_{\Gamma}$ are given by:

$$
q_{m}(z)=\frac{\alpha_{m}}{\sqrt{P_{m m}}} e^{i k_{m} z}
$$

and:

$$
P_{\Gamma_{m n}}=-\frac{i \omega}{4}\left(\mathbf{U}_{\Gamma_{m}}^{*} \mathbf{T}_{\Gamma_{n}}-\mathbf{T}_{\Gamma_{m}}^{*} \mathbf{U}_{\Gamma_{n}}\right)
$$

The modal vector $\mathbf{T}_{m}$ is the so-called modal force associated to the modal displacement of the $m$ th mode and can be readily post-processed from the following formula ${ }^{8,18}$ : $\mathbf{T}_{m}=\left(\mathbf{K}_{2 \sigma}^{T}+i k_{m} \mathbf{K}_{3 \sigma}\right) \mathbf{U}_{m}$.

Expanding Eq. (13) and taking into account the Hermitian property of the matrix $\mathbf{P}_{\Gamma}$ and $k \in \mathbb{R}$ yields the following result:

$$
\Pi_{\Gamma}(z)=\sum_{m=1}^{M} \frac{P_{\Gamma_{m m}}}{P_{m m}}\left|\alpha_{m}\right|^{2}-2 \sum_{m=1}^{M} \sum_{n<m} \operatorname{Re}\left(\frac{P_{\Gamma_{m n}}}{\sqrt{P_{m m} P_{n n}}} \alpha_{m}^{*} \alpha_{n} e^{i\left(k_{n}-k_{m}\right) z}\right)
$$

Now let us consider the case of an integration over the whole cross-section: $\Gamma=S_{0}, \mathbf{U}_{\Gamma}=\mathbf{U}$ and $\mathbf{T}_{\Gamma}=\mathbf{T}$. For conciseness of notations, $\mathbf{P}_{S_{0}}$ will be denoted as $\mathbf{P}$. The notation $P_{m m}$, as used in the previous equations, indeed denotes the power flow of the $m$ th mode integrated over the complete cross-section. The matrix $\mathbf{P}$ obeys the following fundamental property:

$$
\left(k_{m}-k_{n}^{*}\right) P_{m n}=0
$$

Eq. (17) shows that $P_{m n}=0$ if $k_{m} \neq k_{n}^{*}$, i.e. modes $m$ and $n$ are not multiple eigenvalues (note that the complex conjugate of wavenumbers is here extraneous as $k \in \mathbb{R}$ ). As shown in Ref. 23, this property can be viewed as the FE discretized form of Auld's complex biorthogonality relation ${ }^{24}$.

Using Eq. (17) and assuming that no multiple eigenvalues occurs, the last term of Eq. (16) vanishes and the power flow simplifies into the well-known expression:

$$
\Pi=\sum_{m=1}^{M}\left|\alpha_{m}\right|^{2}
$$

The power flow integrated over the whole cross-section is the sum of each individual modal power flows. As expected, it does not depend on $z$ because the power flow of a propagating mode is independent of position along the path of propagation in a lossless medium ${ }^{29,30}$.

\section{Energy transfer parameter}

One is interested in the transfer of energy from a given wire, directly excited, to the other wires, only excited through interwire contact. The former will be referred to as the active wire. The latter will be called the passive wires. Thus, one considers an excitation localized inside a specified wire and investigates how the energy transfers from this wire to the others. We define the energy transfer parameter $\eta$, equal to the ratio of the power flow inside the six passive wires to the power flow of the whole seven-wire strand:

$$
\eta(z)=1-\frac{\Pi_{\Gamma}(z)}{\Pi}
$$

where $\Gamma$ will now denote the cross-section of the active wire in the remainder. A small value of $\eta$ thus means a weak energy transfer from the active wire to the others and conversely. Typically, the parameter $\eta$ will be equal to:

- 0 when the energy remains totally confined inside the active wire;

- 1 when there is no energy inside the active wire;

- $6 / 7 \simeq 0.86$ if there is equipartition of energy inside the whole strand;

- $5 / 6 \simeq 0.83$ if, in the case of an active peripheral wire, there is equipartition of energy inside the six peripheral wires and no energy inside the central one. 


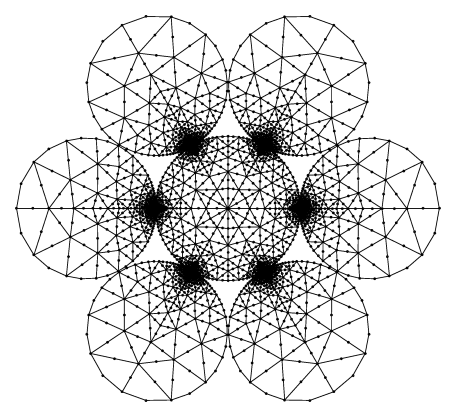

FIG. 2: Cross-section FE mesh of the seven-wire strand.

As opposed to the power flow $\Pi$ (integrated over the whole cross-section $S_{0}$ ), the last term of $\Pi_{\Gamma}$ in Eq. (16) does not vanish for a substructure constituting the complete waveguide because Auld's biorthogonality relation (17) only holds for the complete system. This term depends on $z$, and as a consequence, so does the energy transfer parameter $\eta$.

Yet, it can be noticed that the $z$ dependence of the last term in (16) is in $e^{i\left(k_{n}-k_{m}\right) z}$, corresponding to an oscillating term whose average along the $z$ direction vanishes. In order to simplify the analysis of energy transfer, we define in this paper the $z$-averaged energy transfer parameter, denoted as $\bar{\eta}$, given by:

$$
\bar{\eta}=1-\frac{\bar{\Pi}_{\Gamma}}{\Pi}
$$

with the $z$-averaged power flow through the surface $\Gamma$ :

$$
\bar{\Pi}_{\Gamma}=\sum_{m=1}^{M} \frac{P_{\Gamma_{m m}}}{P_{m m}}\left|\alpha_{m}\right|^{2}
$$

\section{RESULTS}

\section{A. Preliminary results: free response}

Computations are performed for the following parameters: $r_{p} / r_{c}=0.967, \phi=7.9^{\circ}, \nu=0.28 . \quad r_{c}$ and $r_{p}$ denote the radius of the central and peripheral wire respectively. The helix lay angle $\phi$ is defined from the relation $\tan \phi=2 \pi\left(r_{c}+r_{p}\right) / L_{i}$ and the torsion of the twisting system is $\tau_{i}=2 \pi / L_{i}$, with $L_{i}$ denoting the length at rest of one helix pitch along the $z$-axis of peripheral wires. Normalized results will be presented. When specified, some dimensional results will be provided with: $r_{c}=2.7 \mathrm{~mm}, E=2.17 \mathrm{e} 11 \mathrm{~Pa}, \rho=7800 \mathrm{~kg} / \mathrm{m}^{3}$ (material properties of steel).

The cross-section of the seven-wire strand has been meshed with $\mathrm{Gmsh}^{31}$ and is shown in Fig. 2. Six-node triangles are used yielding 12369 dofs. The mesh is refined near contact regions.

Before considering the interwire energy transfer of the response under excitation, the main results found for the free response in previous works ${ }^{6,8}$ must be recalled.

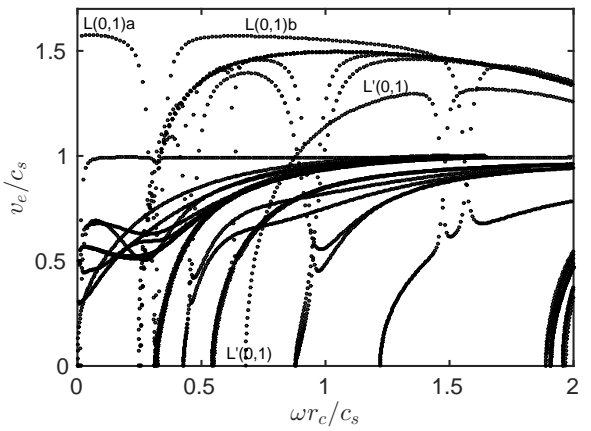

(a)

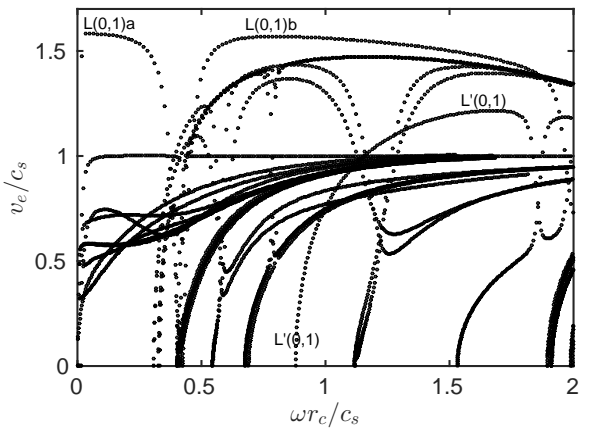

(b)

FIG. 3: Dispersion curves of the unloaded seven-wire strand, (a) unloaded $(\epsilon=0)$ and (b) loaded $(\epsilon=0.6 \%)$.

Figure 3 shows the normalized energy velocity dispersion curves for the seven-wire strand, unloaded and subjected to an axial strain of $\epsilon=0.6 \%$. The normalized frequency is given by $\omega r_{c} / c_{s}$, with $c_{s}=\sqrt{E / 2 \rho(1+\nu)}$ denoting the shear wave velocity. As for the static computation, 13 contact node pairs were necessary for each interwire region.

Due to the multi-wire nature of the waveguide, many modes are found compared to a single wire, for which only three modes are propagating in the frequency range ${ }^{6}$ (these modes are the first longitudinal, torsional and flexural modes, often labeled $L(0,1), T(0,1)$ and $F(1,1)$ respectively).

A particular phenomenon occurs for the first longitudinal mode. Instead of a single continuous curve in a single wire, this mode splits into two curves in a sevenwire strand, denoted as $L(0,1) a$ and $L(0,1) b$ in Fig. 3 . This split indeed corresponds to a curve veering phenomenon ${ }^{6}$. This veering phenomenon is centered around the frequency $\omega r_{c} / c_{s}=0.32$ for $\epsilon=0$, increasing to 0.42 for $\epsilon=0.6 \%$.

\section{B. Note on model validation}

The implementation of the SAFE method, in twisting coordinates and with prestress, has been checked in 
Ref. 8 for springs, that is to say single wires of large helix angle subjected to large elongation. Close agreement has been found between the numerical dispersion curves and results obtained from a Timoshenko helical beam approximation.

In addition to twisting and prestress effects, the case of seven-wire strands leads to another difficulty: the mechanics of interwire contact must be included in the analysis. The fundamental assumption used in the SAFE model is infinite friction (perfectly stick contact conditions). This assumption has shown to give satisfying results for the so-called notch frequency, experimentally observed in the literature. As previously mentioned, the normalized curve veering frequency obtained from the SAFE model significantly increases, roughly from 0.32 for $\epsilon=0$ to 0.42 for $\epsilon=0.6 \%$ (in this paper, this yields dimensional frequency of $62 \mathrm{kHz}$ and $82 \mathrm{kHz}$ respectively). These values are in agreement with the notch frequency of experiments ${ }^{1,2,6}$. This provides a preliminary experimental validation of the model.

More precisely, numerical tests have shown that a loaded strand model neglecting contact, i.e. with only one contact node pair at each interwire region, leaves the curve veering frequency of the unloaded case unchanged ${ }^{8}$. This means that the increase of the notch frequency is mainly caused by the increase of the interwire contact width, rather than prestress itself. Yet, the computation of the contact width has not been checked. Its validation turns out to be necessary and is performed in the present paper.

To this end, both the contact half-width $a$ and the normal contact force $N_{0}$ are post-processed from the numerical solution computed from Sec. II C when the applied strain varies from $\epsilon=0$ to $\epsilon=0.6 \%$. Figure 4 shows $a$ as a function of $N_{0}$ computed from the model as well as the theoretical solution obtained from Hertz theory for parallel cylinders ${ }^{32}$. Close agreement is found, which validates the contact iterative process described in Sec. II C. Note that one can conclude that the effect of helical geometry on the contact width is almost negligible here. However, accounting for the helical geometry is essential for the computation of $N_{0}$ (if parallel wires were considered, the contact force would remain equal to zero).

Further experimental works will be needed to assess the limitations of the stick contact assumption in the SAFE model. For a given displacement amplitude, the acoustic stress tends to increase with frequency. In the interwire region, an increase of the shear stress may lead to imperfect contact involving stick-slip phenomena and friction. A perfect contact assumption implies that the acoustic shear stress is small enough in the contact width, that is to say below the normal stress times the friction coefficient from Coulomb's law. In practice, tensile loads in cables are huge and lead to large static contact stresses in the normal direction. These static contact stresses can be assumed far greater than acoustic shear stresses, at least up to a certain frequency limit, so that perfectly stick contact conditions appear reasonable as a first ap-

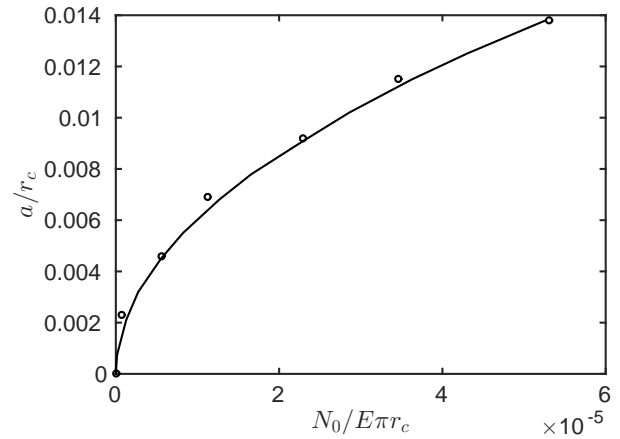

FIG. 4: Normalized contact half-width as a function of the normal contact force. Circles: numerical results, continuous lines: Hertz solution.

proximation.

In the static model, the applied axial strain of $0.6 \%$ gives a tension of $190 \mathrm{kN}$, that is to say a mean axial stress of $1.26 \mathrm{GPa}$ in the cross-section of the strand. This stress level corresponds an operational load of about $60 \%$ of the ultimate tensile strength, as considered in experiments $^{1,2,13}$. From Fig. 4 at the maximum load $(\epsilon=0.6 \%)$, the dimensional static normal force $N_{0}$ at each interwire contact is equal to $97.7 \mathrm{kN} / \mathrm{m}$, that is to say a mean normal stress of $1.32 \mathrm{GPa}$ (the contact width $2 a$ being equal to $74 \mu \mathrm{m})$. As far as the tangential component of the static contact force is concerned, the model gives a value that remains small compared to the normal component.

\section{Results for longitudinal excitation}

One considers an excitation $\mathbf{F}(z)$, of volume type, localized inside the central wire. Peripheral wires are not excited. The excitation is oriented along the guiding direction in order to mainly excite compressional-like modes. The excitation profile is distributed over the cross-section of the active wire as a radial cosine function vanishing at its boundary, as depicted in Fig. 5. One yet emphasized that the results presented in this paper are little sensitive to the excitation profile (actually, using point source excitations instead yields nearly identical results).

The excitation is concentrated at $z=0$, which means that its $z$ dependence is the Dirac function, so that the space Fourier transform of the excitation $\tilde{\mathbf{F}}(k)$ does not depend on $k$. The excitation is assumed to be constant with frequency. In the remainder of this paper, only the loaded case will be considered $(\epsilon=0.6 \%)$. The unloaded case is somehow purely theoretical since the contact width tends to zero (only one interwire contact node pair).

Figure 6a shows the averaged energy transfer parameter $\bar{\eta}$ as a function of frequency for a loaded strand $(\epsilon=0.6 \%)$. As expected, the energy transfer to peripheral 


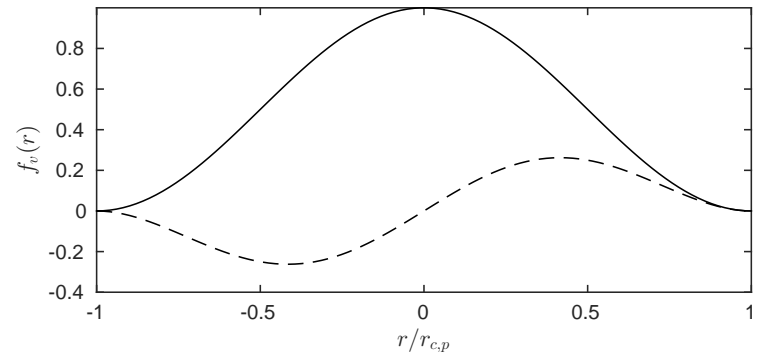

FIG. 5: Excitation profiles as a function of the local radial coordinate of the active wire. Continuous line: excitation of longitudinal and flexural type (oriented in the $z$ and $x$ direction respectively). Dashed line: excitation of torsional type (oriented in the local azimuthal direction).

wires is high in a low frequency range $\left(\omega r_{c} / c_{s} \in[0 ; 1]\right)$. This is due to the global behavior of the $L(0,1)$-like mode in this frequency range: the strand moves as a whole in the longitudinal direction ${ }^{6}$. This global motion is confirmed by a value of $\bar{\eta}$ close to $6 / 7$, due to equipartition of energy inside wires. Note that a local decrease of $\bar{\eta}$ can be observed around the veering frequency owing to the sudden change of mode shapes associated to the $L(0,1)$ like modes.

In the frequency range $[1 ; 2]$, the energy transfer becomes weak with a sharp decrease of $\bar{\eta}$ for $\omega r_{c} / c_{s} \sim 0.88$. This suggests that the motion of the strand tends to be localized inside the central wire. This sudden change is attributed to the excitation of another compressional-like mode, denoted as $L^{\prime}(0,1)$, whose motion is localized in the central wire. This particular mode is identified in Fig. 3. Its cut-off frequency occurs at $\omega r_{c} / c_{s}=0.88$, which coincides with the sudden drop of energy transfer. Figure $6 \mathrm{~b}$ plots the modal coefficient modulus of all propagating modes. It can be seen that the amplitude of the localized $L^{\prime}(0,1)$ mode after its cut-on is clearly greater than the other modes. Figure 7 shows the mode shape of the $L^{\prime}(0,1)$ mode at the frequency $\omega r_{c} / c_{s}=1.5$, which confirms that this mode implies a negligible motion of peripheral wires.

This type of local mode has also been found in the numerical investigation of dispersion curves of strands embedded into a solid matrix ${ }^{33}$. Due to weak motion in peripheral wires, such a local mode is less sensitive to the loss induced by the exterior medium and can be of potential interest for NDE and structural health monitoring applications. As can be observed from Fig. 3, another interesting feature of this mode could be its sensitivity to contact, and hereby to applied loads.

It should be mentioned that the transfer of energy from the central wire to the peripheral ones has also been found to decrease with frequency in experiments ${ }^{34}$.

Figure 8 illustrates the dependence of the power flow of a substructure by plotting the non-averaged energy transfer parameter $\eta(z)$ as a function of the propaga-

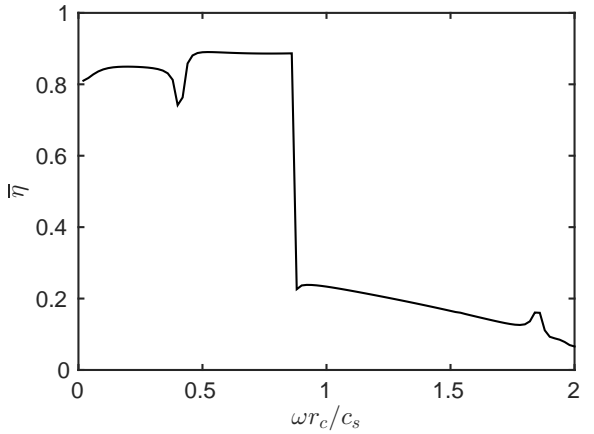

(a)

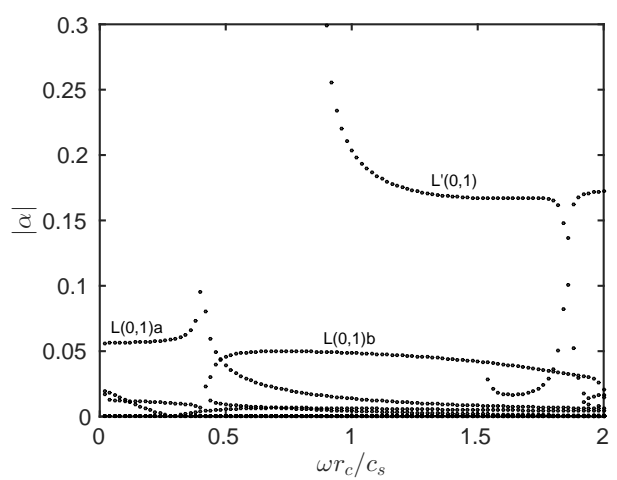

(b)

FIG. 6: (a) Averaged energy transfer parameter $\bar{\eta}$ for an excitation localized in the central wire and (b) modulus of modal coefficients $\left|\alpha_{m}\right|$ as a function of the normalized frequency for a strand subjected to a $0.6 \%$ tensile strain.

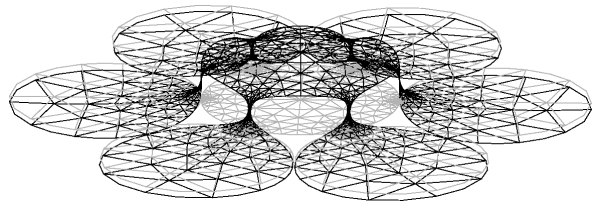

FIG. 7: Mode shape of the $L^{\prime}(0,1)$ mode for $\omega r_{c} / c_{s}=1.5$.

tion distance, the frequency being set to a fixed value $\omega r_{c} / c_{s}=1.5$. The value of $\eta(z)$ oscillates around an averaged value $\bar{\eta} \simeq 0.16$. These oscillations occur with nonnegligible amplitudes but the energy transfer remains low.

Let us now consider a longitudinal-like excitation localized inside a peripheral wire. No matter which peripheral wire is excited due to the rotational symmetry of the geometry. Figure 9a exhibits the averaged energy transfer parameter $\bar{\eta}$. The value of $\bar{\eta}$ is nearly constant and large over the whole frequency range, which means that the energy does not remain localized inside the active wire as the waves propagate along the axis.

Indeed, the non-averaged parameter $\eta(z)$ can strongly 


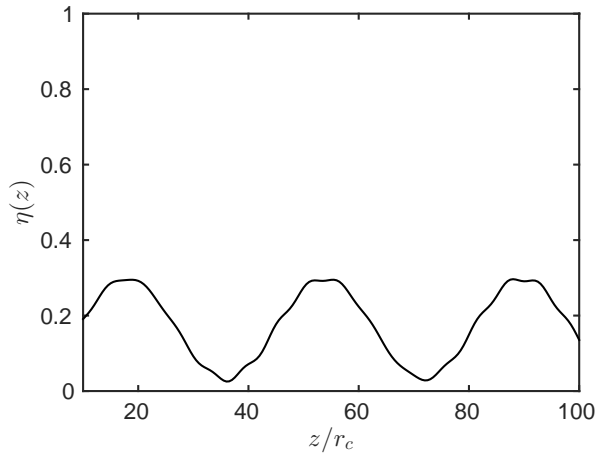

FIG. 8: Non-averaged energy transfer parameter $\eta(z)$ as a function of the propagation distance $z / r_{c}$ for $\omega r_{c} / c_{s}=1.5$.

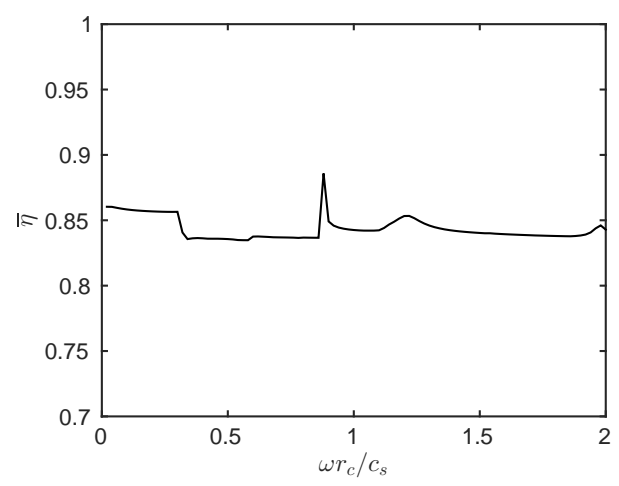

(a)

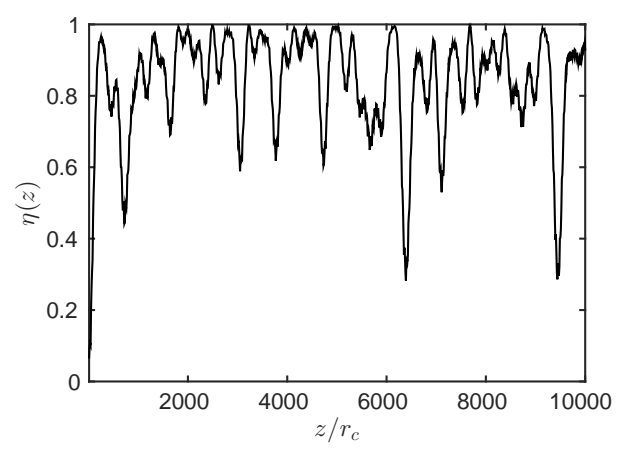

(b)

FIG. 9: (a) Averaged energy transfer parameter $\bar{\eta}$ for an excitation localized in a peripheral wire, (b) $\eta(z)$ for $\omega r_{c} / c_{s}=1.5$.

differs from its averaged value. As an example, $\eta(z)$ is plotted in Fig. $9 \mathrm{~b}$ for the frequency $\omega r_{c} / c_{s}=1.5$. This means that the strand response strongly depends on the propagation distance $z$ from the source, as clearly illustrated by Fig. 10. Depending on $z$, the response can be localized in the active peripheral wire (for instance, at $z / r_{c}=50$, which is close to the source), spread over different wires at greater distance, or even be localized into

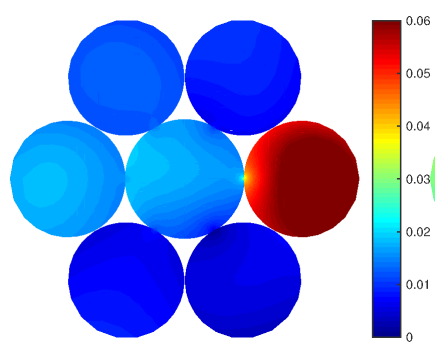

(a)

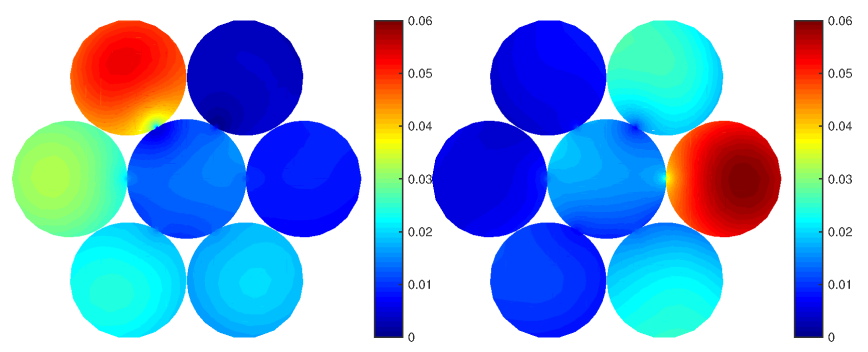

(c)

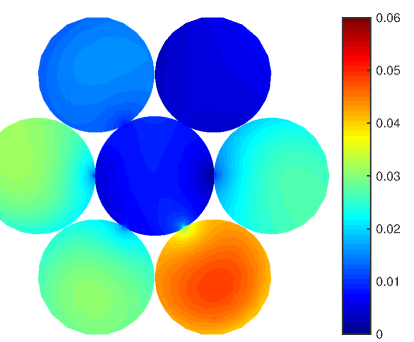

(b)
FIG. 10: Modulus of the axial displacement field excited by a peripheral wire source for $\omega r_{c} / c_{s}=1.5$ at: (a) $z / r_{c}=50(\eta=0.21),(\mathrm{b}) z / r_{c}=1000(\eta=0.87)$, (c) $z / r_{c}=2000(\eta=0.98)$, (d) $z=6400(\eta=0.34)$ (Color online).

passive wires only.

This phenomenon confirms and highlights some experimental results of the literature ${ }^{35}$ found on the energy transfer variation with respect to the actuator distance when exciting a peripheral wire. In the context of NDE, such a phenomenon is likely to complicate the interpretation of measurement as well as the identification of damaged wires, as experimentally observed in Ref. 36 .

Figure 11a gives further insight by plotting colored dispersion curves indicating modal amplitudes $\left|\alpha_{m}\right|$ for a peripheral excitation. Up to a normalized frequency roughly equal to 0.3 , the excited mode is mainly the $L(0,1)$ a mode. The motion is hence of global type and $\bar{\eta}$ is close to $6 / 7$ (see Fig. 9a). Then above this frequency, the motion changes due to the cut-on of several modes of longitudinal type (Fig. 11a), which most contribute to the response. These modes are mainly peripheral and involve a weak motion of the central wire. The value of $\eta$ thus tends to $5 / 6$ because the motion of the central wire becomes negligible. These peripheral modes are quite close to each other and strongly combine under excitation in the forced response: this multimodal propagation gives rise to a significant oscillatory contribution in Eq. (16)(see second term of the expansion of $\Pi_{\Gamma}(z)$ ).

Figure 11a can be compared to Fig. 11b, obtained for the excitation of the central wire. In the latter case, the modal contribution of the group of cut-on peripheral modes observed earlier is negligible.

As observed from Figs. 9a and 10, note that a large value of $\bar{\eta}$ does not necessarily imply a global motion of 


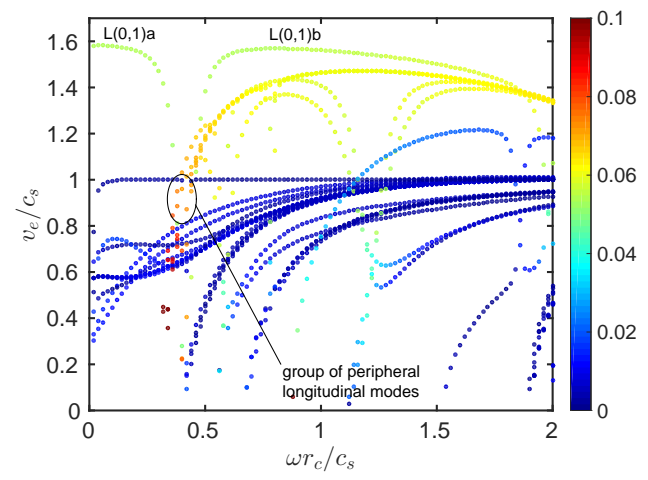

(a)

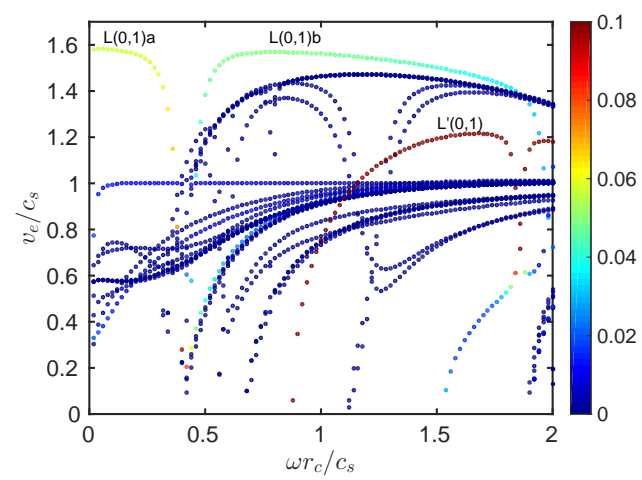

(b)

FIG. 11: Energy velocity dispersion curves with their modal amplitude for the excitation of (a) a peripheral wire and (b) the central wire (color online).

the strand: a large energy transfer can also occur with a multimodal propagation involving a group of non global modes.

\section{Other types of excitation}

Figure 12 presents the results obtained with an excitation of flexural type localized inside the central wire (see profile in Fig. 5). The excitation has been oriented along the $x$-direction but changing the polarization to the $y$-direction almost does not change the results.

Up to $\omega r_{c} / c_{s} \sim 0.54$, the motion is global $(\bar{\eta}$ is close to $6 / 7)$. The mode labeled as $F(1,1)$ is strongly excited and behaves as a global flexural modes by analogy with cylinders. The normalized frequency of 0.54 coincides with the cut-on of two flexural modes, labeled as $F^{\prime}(1,1)$, that tend to be localized in the central wire as the frequency increases. Then from $\omega r_{c} / c_{s} \sim 0.6$, the energy parameter starts to decrease. Nevertheless, the non averaged value of $\eta(z)$ oscillates with strong amplitude, even for the highest frequency $\left(\omega r_{c} / c_{s}=2\right)$ as shown by Fig. 13a, for the same reasons as for the longitudinal excitation of a peripheral wire (i.e. multimodal propagation of a group

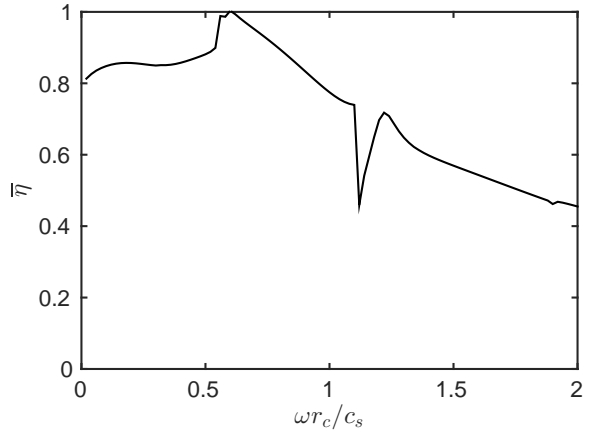

(a)

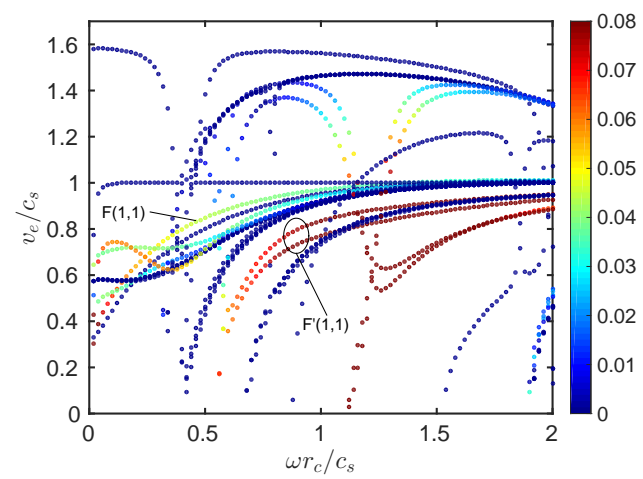

(b)

FIG. 12: (a) Averaged energy transfer parameter $\bar{\eta}$ for a flexural-like excitation localized in the central wire, (b) energy velocity curves with modal amplitudes (color online).

of non-global modes). This phenomenon is also likely to explain the variation with distance of the $F(1,1)$ transmission ratio observed in some experiments ${ }^{15}$. Hence, no clear localization in the central wire occurs and the energy travels in every wire as the waves propagate in the $z$-direction.

Around the normalized frequency of 0.6 , it can be observed that the value of $\bar{\eta}$ is nearly equal to 1 . This could a priori mean that the whole energy has been transferred to peripheral wires at this particular frequency. However, it should be recalled that the power flow, while positive when integrated over the whole cross-section, can possibly be negative in some subregion. Figure 13b depicts the non averaged energy transfer parameter $\bar{\eta}$ as a function of $z$ for $\omega r_{c} / c_{s}=0.6$. Depending on the distance, its value can be slightly greater than one, which implies that the power flow inside the central wire can be weakly negative. Similar reverse energy fluxes occurs in stratified waveguides $^{37}$ for instance.

Finally, Fig. 14 shows results obtained for an excitation of torsional type (see dashed line profile in Fig. 5) localized inside the central wire. The excitation is oriented along the angular direction of the wire in order mainly excite torsional modes. In this case, the interpretation 


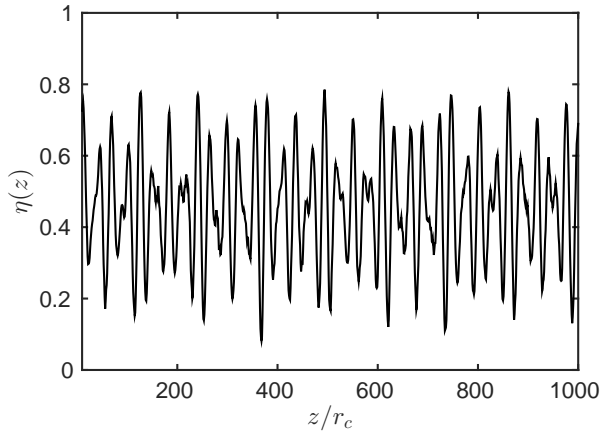

(a)

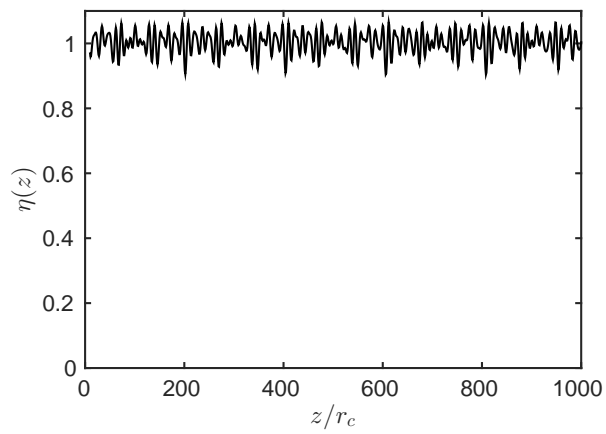

(b)

FIG. 13: Non-averaged energy transfer parameter as a function of the propagation distance $z / r_{c}$ for a flexural-like excitation localized in the central wire for (a) $\omega r_{c} / c_{s}=2$, (b) $\omega r_{c} / c_{s}=0.6$.

of results is similar to the longitudinal excitation of central wire. Up to a certain frequency, a global motion is observed corresponding to the global torsional mode, denoted as $T(0,1)$ by analogy with cylindrical bars $(\bar{\eta}$ tends to $6 / 7)$. Here, this frequency is roughly equal to 1.5 and coincides with the cut-off frequency of a torsional mode of local type, confined in the central wire and labeled as $T^{\prime}(0,1)$. However, this localization of torsional type appears to be of less interest for NDE purpose because the $T^{\prime}(0,1)$ mode is quite dispersive (see Fig. 14b) in the frequency range where localization occurs.

As for the excitation of peripheral wires (results not shown for conciseness), their excitation based on a flexural or torsional polarization leads to the same kind of results and interpretations as those obtained with a longitudinal excitation: no localization is observed, the value of $\bar{\eta}$ remains quite large (greater than 0.8 ) over the whole frequency range.

\section{CONCLUSION}

In this paper, the transfer of energy inside a multiwire cable has been investigated with a SAFE method. The forced response of the structure has been calcu-

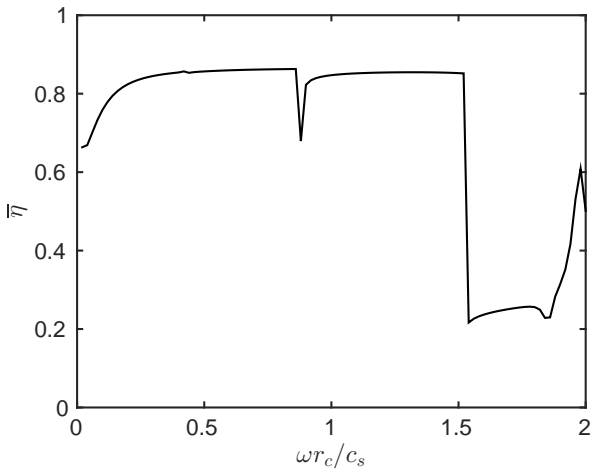

(a)

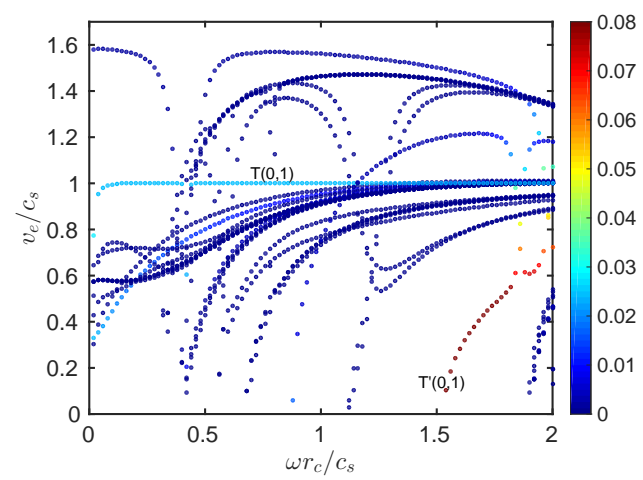

(b)

FIG. 14: (a) Averaged energy transfer parameter $\bar{\eta}$ for a torsional-like excitation localized in the central wire, (b) energy velocity curves with modal amplitudes (color online).

lated thanks to a modal approach and a biorthogonality relation. This biorthogonality relation indeed corresponds to a discretized version of Auld's complex relation, which turns out to extend both to twisted and prestressed waveguides.

An energy transfer parameter has been proposed in order to simplify the interpretation of the power flow distribution inside the cable and the exchange of energy between wires. This parameter is equal to the ratio of the $z$-averaged power flow of passive wires to the total power flow.

Based on this parameter, a compressional mode mainly localized inside the central wire has been found. This mode can be strongly excited with an axial excitation of the central wire. This type of local mode could be of interest for NDE applications. In the frequency range considered, no similar localization has been found with an active peripheral wire.

It has also been shown that the strand response and its energy distribution generally strongly depend on the propagation distance from the source. Due to multimodal propagation phenomena, the energy inside a specific wire may indeed strongly oscillate along the axis of 
the waveguide. Depending on the distance, the response can be localized inside the active wire, spread over one or several wires at greater distance, or even be localized into passive wires only. Such a phenomenon complicates the interpretation of measurements and the analysis of guided wave scattering by defects, and is likely to highlight experimental results available in the literature.

Owing to the complex pattern of dispersion curves inside multi-wire structures, the analysis of the forced response under several polarizations and locations of excitation helps to identify the nature of modes (longitudinal, flexural or torsional).

Further works should deal with experiments to highlight the effects of interwire stick-slip and friction phenomena, neglected in the present numerical model.

\section{Appendix A: Expressions of FE matrices}

The element matrices involved in Eq. (3) are given by:

$$
\begin{array}{r}
\mathbf{M}^{e}=\int_{S_{0}^{e}} \rho_{0} \mathbf{N}^{e T} \mathbf{N}^{e} d x d y \\
\mathbf{K}_{1 \sigma}^{e}=\mathbf{K}_{1}^{e}+\int_{S_{0}^{e}} \mathbf{N}^{e T} \mathbf{G}_{x y}^{T} \boldsymbol{\Sigma}_{0} \mathbf{G}_{x y} \mathbf{N}^{e} d x d y \\
\mathbf{K}_{2 \sigma}^{e}=\mathbf{K}_{2}^{e}+\int_{S_{0}^{e}} \mathbf{N}^{e T} \mathbf{G}_{x y}^{T} \mathbf{\Sigma}_{0} \mathbf{G}_{z} \mathbf{N}^{e} d x d y \\
\mathbf{K}_{3 \sigma}^{e}=\mathbf{K}_{3}^{e}+\int_{S_{0}^{e}} \mathbf{N}^{e T} \mathbf{G}_{z}^{T} \mathbf{\Sigma}_{0} \mathbf{G}_{z} \mathbf{N}^{e} d x d y \\
\mathbf{K}_{1}^{e}=\int_{S_{0}^{e}} \mathbf{N}^{e T} \mathbf{L}_{x y}^{T} \mathbf{C}_{0} \mathbf{L}_{x y} \mathbf{N}^{e} d x d y \\
\mathbf{K}_{2}^{e}=\int_{S_{0}^{e}} \mathbf{N}^{e T} \mathbf{L}_{x y}^{T} \mathbf{C}_{0} \mathbf{L}_{z} \mathbf{N}^{e} d x d y \\
\mathbf{K}_{3}^{e}=\int_{S_{0}^{e}} \mathbf{N}^{e T} \mathbf{L}_{z}^{T} \mathbf{C}_{0} \mathbf{L}_{z} \mathbf{N}^{e} d x d y .
\end{array}
$$

The matrix $\mathbf{C}_{0}$ denotes the matrix of elastic properties relating stress and strain in their vector form by $\boldsymbol{\sigma}=$ $\mathbf{C}_{0} \boldsymbol{\epsilon}$, with: $\boldsymbol{\epsilon}=\left[\begin{array}{llllll}\epsilon_{x x} & \epsilon_{y y} & \epsilon_{z z} & 2 \epsilon_{x y} & 2 \epsilon_{x z} & 2 \epsilon_{y z}\end{array}\right]^{T}$ and $\boldsymbol{\sigma}=$

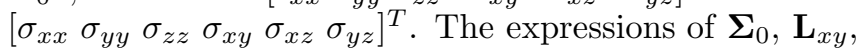

$\mathbf{L}_{z}, \mathbf{G}_{x y}$ and $\mathbf{G}_{z}$ are given by:

$$
\begin{array}{r}
\mathbf{\Sigma}_{0}=\left[\begin{array}{ccc}
\sigma_{0} & \mathbf{0} & \mathbf{0} \\
\mathbf{0} & \sigma_{0} & \mathbf{0} \\
\mathbf{0} & \mathbf{0} & \sigma_{0}
\end{array}\right], \\
\mathbf{L}_{x y}=\left[\begin{array}{ccc}
\partial / \partial x & 0 & 0 \\
0 & \partial / \partial y & 0 \\
0 & 0 & \Lambda \\
\partial / \partial y & \partial / \partial x & 0 \\
\Lambda & -\tau_{0} & \partial / \partial x \\
\tau_{0} & \Lambda & \partial / \partial y
\end{array}\right], \mathbf{L}_{z}=\left[\begin{array}{ccc}
0 & 0 & 0 \\
0 & 0 & 0 \\
0 & 0 & 1 \\
0 & 0 & 0 \\
1 & 0 & 0 \\
0 & 1 & 0
\end{array}\right], \\
\mathbf{G}_{x y}=\left[\begin{array}{ccc}
\partial / \partial x & 0 & 0 \\
\partial / \partial y & 0 & 0 \\
\Lambda & -\tau_{0} & 0 \\
0 & \partial / \partial x & 0 \\
0 & \partial / \partial y & 0 \\
\tau_{0} & \Lambda & 0 \\
0 & 0 & \partial / \partial x \\
0 & 0 & \partial / \partial y \\
0 & 0 & \Lambda
\end{array}\right], \mathbf{G}_{z}=\left[\begin{array}{lll}
0 & 0 & 0 \\
0 & 0 & 0 \\
1 & 0 & 0 \\
0 & 0 & 0 \\
0 & 0 & 0 \\
0 & 1 & 0 \\
0 & 0 & 0 \\
0 & 0 & 0 \\
0 & 0 & 1
\end{array}\right],
\end{array}
$$

where $\Lambda=\tau_{0}(y \partial / \partial x-x \partial / \partial y)$. In Eqs. (A1), the integral terms in $\boldsymbol{\Sigma}_{0}$ correspond to the so-called geometric stiffness, related to the presence of a prestress field $\left(\sigma_{0} \neq \mathbf{0}\right)$.

Note that the element matrices (A1) must be expressed on the prestressed cross-section $S_{0}$. In practice, this means that the initial FE mesh should be updated to the prestressed geometry before the computation of SAFE matrices. Under the assumption of small strain, which is used in this paper, mechanical properties can be considered as independent of strain so that the following equalities hold ${ }^{19}: \mathbf{C}_{0}=\mathbf{C}_{i}$ and $\rho_{0}=\rho_{i}$, where the subscript $i$ refers to the initial configuration (undeformed, unprestressed).

The element matrix and vector involved in the static computation of the prestressed state (Sec. II C) are:

$$
\begin{array}{r}
\mathbf{K}_{0}^{e}=\int_{S_{i}^{e}} \mathbf{N}^{e T} \mathbf{L}_{x y}^{T} \mathbf{C}_{i} \mathbf{L}_{x y} \mathbf{N}^{e} d x d y \\
\mathbf{F}_{0}^{e}=-\int_{S_{i}^{e}} \mathbf{N}^{e T} \mathbf{L}_{x y}^{T} \mathbf{C}_{i} \boldsymbol{\epsilon}_{M} d x d y
\end{array}
$$

where $\boldsymbol{\epsilon}_{M}=\left[\begin{array}{llllll}0 & 0 & \epsilon & 0 & 0 & 0\end{array}\right]^{T}$. Note that $\mathbf{K}_{0}$ and $\mathbf{F}_{0}$ are integrated on $S_{i}$ (undeformed cross-section). In these expressions, the operator $\mathbf{L}_{x y}$ should be understood as the expression given in Eq. (A2) replacing $\tau_{0}$ with $\tau_{i}$, the torsion of the undeformed geometry. The torsions $\tau_{i}$ and $\tau_{0}$ are linked by: $\tau_{0}=\tau_{i} /(1+\epsilon)$.

${ }^{1}$ H. Kwun, K. A. Bartels, and J. J. Hanley, "Effects of tensile loading on the properties of elastic-wave propagation in a strand," Journal of the Acoustical Society of America 103, 3370-3375 (1998).

${ }^{2}$ L. Laguerre, M. Brissaud, and J. C. Aime, "Low-frequency ultrasound reflectometry device based on magnetoelastic transducers for the non destructive evaluation of steel rods and cables," Bulletin des Laboratoires des Ponts et Chaussées 239, 7-27 (2002). ${ }^{3}$ P. Rizzo and F. L. di Scalea, "Load measurement and health monitoring in cable stays via guided wave magnetostrictive ultrasonics," Materials Evaluation 62, 1057-1065 (2004). 
${ }^{4} \mathrm{~F}$. Treyssède, "Numerical investigation of elastic modes of propagation in helical waveguides," Journal of the Acoustical Society of America 121, 3398-3408 (2007).

${ }^{5} \mathrm{~F}$. Treyssède, "Elastic waves in helical waveguides," Wave Motion 45, 457-470 (2008).

${ }^{6} \mathrm{~F}$. Treyssède and L. Laguerre, "Investigation of elastic modes propagating in multi-wire helical waveguides," Journal of Sound and Vibration 329, 1702-1716 (2010).

${ }^{7}$ A. Frikha, P. Cartraud, and F. Treyssède, "Mechanical modeling of helical structures accounting for translational invariance. part 1: Static behavior," International Journal of Solids and Structure 50, 1373-1382 (2013).

${ }^{8}$ F. Treyssède, A. Frikha, and P. Cartraud, "Mechanical modeling of helical structures accounting for translational invariance. part 2: Guided wave propagation under axial loads," International Journal of Solids and Structure 50, 1383-1393 (2013).

${ }^{9}$ L. Gavric, "Computation of propagative waves in free rail using a finite element technique," Journal of Sound and Vibration 185, 531-543 (1995).

${ }^{10}$ T. Hayashi, W.-J. Song, and J. L. Rose, "Guided wave dispersion curves for a bar with an arbitrary cross-section, a rod and rail example," Ultrasonics 41, 175-183 (2003).

${ }^{11}$ M. Mazzotti, A. Marzani, I. Bartoli, and E. Viola, "Guided waves dispersion analysis for prestressed viscoelastic waveguides by means of the SAFE method," International Journal of Solids and Structure 49, 2359-2372 (2012).

${ }^{12}$ M. Mazzotti, I. Bartoli, and A. Marzani, "Ultrasonic leaky guided waves in fluid-coupled generic waveguides: hybrid finiteboundary element dispersion analysis and experimental validation," Journal of Applied Physics 115, 143512 (2014).

${ }^{13}$ I. Bartoli, S. Salamone, R. Phillips, F. L. di Scalea, and C. S. Sikorsky, "Use of interwire ultrasonic leakage to quantify loss of prestress in multiwire tendons," Journal of Engineering Mechanics 137, 324-333 (2011).

${ }^{14}$ C. Nucera and F. L. di Scalea, "Monitoring load levels in multiwire strands by nonlinear ultrasonic waves," Structural Health Monitoring 106, 617-629 (2011).

${ }^{15}$ R. Raisutis, R. Kazys, L. Mazeika, E. Zukauskas, V. Samaitis, and A. Jankauskas, "Ultrasonic guided wave-based testing technique for inspetcion of multi-wire rope structures," NDT\&E International 62, 40-49 (2014).

${ }^{16}$ J. Schotté and R. Ohayon, "Incompressible hydroelastic vibrations: finite element modeling of the elastogravity operator," Computers \& Structures 83, 209-219 (2005).

${ }^{17} \mathrm{~F}$. Treyssède and M. B. Tahar, "Jump conditions for unsteady small perturbations at fluidsolid interfaces in the presence of initial flow and prestress," Wave Motion 46, 155-167 (2009).

${ }^{18} \mathrm{~F}$. Treyssède, "Mode propagation in curved waveguides and scattering by inhomogeneities: application to the elastodynamics of helical structures," Journal of the Acoustical Society of America 129, 1857-1868 (2011).

${ }^{19}$ K. J. Bathe, Finite Element Procedures (Prentice Hall, Englewood Cliffs, New Jersey, 1996) 1037 p.

${ }^{20} \mathrm{P}$. Wriggers, Computational Contact Mechanics (Springer-
Verlag, Berlin) 518 p.

${ }^{21} \mathrm{~F}$. Tisseur and $\mathrm{K}$. Meerbergen, "The quadratic eigenvalue problem," SIAM Review 43, 235-286 (2001).

${ }^{22} \mathrm{~V}$. Baronian, Coupling of modal and finite elements methods for the diffraction of guided elastic waves - Application to Non Destructive Testing (in French), Ph.D. thesis, Ecole Polytechnique (2009).

${ }^{23} \mathrm{~F}$. Treyssède and L. Laguerre, "Numerical and analytical calculation of modal excitability for elastic wave generation in lossy waveguides," Journal of the Acoustical Society of America 133, 3827-3837 (2013).

${ }^{24}$ B. A. Auld, Acoustic Fields and Waves in Solids, 2nd ed., Vol. II (Krieger, Malabar, FL, 1990) 432 p.

${ }^{25}$ W. B. Fraser, "Othogonality relation for rayleigh-lamb modes of vibration of a plate," Journal of the Acoustical Society of America 59, 215-216 (1976).

${ }^{26} \mathrm{~V}$. Pagneux and A. Maurel, "Lamb wave propagation in elastic waveguides with variable thickness," Proceedings of the Royal Society A 462, 1315-1339 (2006).

${ }^{27}$ W. B. Fraser, "Separable equations for a cylindrical anisotropic elastic waveguide," Journal of Sound and Vibration 72, 151-157 (1980).

${ }^{28}$ F. Benmeddour, F. Treyssède, and L. Laguerre, "Numerical modeling of guided waves interaction with non-axisymmetric cracks in elastic cylinders," International Journal of Solids and Structures 48, 764-774 (2011).

${ }^{29}$ B. A. Auld, Acoustic Fields and Waves in Solids, 2nd ed., Vol. I (Krieger, Malabar, FL, 1990) 446 p.

${ }^{30}$ R. E. Collin, Field Theory of Guided Waves (IEEE Press, 1991) $864 \mathrm{p}$.

${ }^{31}$ C. Geuzaine and J.-F. Remacle, "Gmsh: a three-dimensional finite element mesh generator with built-in pre- and postprocessing facilities," International Journal for Numerical Methods in Engineering 79, 1309-1331 (2009).

${ }^{32}$ N. Chandrasekaran, W. E. Haisler, and R. E. Goforth, "Finite element analysis of Hertz contact problem with friction," Finite Elements in Analysis and Design 3, 39-56 (1987).

${ }^{33}$ K. L. Nguyen and F. Treyssède, "Numerical investigation of leaky modes in helical structural waveguides embedded into a solid medium," Ultrasonics 57, 127-134 (2015).

${ }^{34}$ I. Bartoli, A. Marzani, F. L. di Scalea, P. Rizzo, E. Viola, E. Sorrivi, and R. Phillips, "SAFE modeling of waves for the structural health monitoring of prestressing tendons," in Proc. of SPIE Vol. 6532, Health Monitoring of Structural and Biological Systems 2007 (2007) pp. 65320D1-65320D12.

${ }^{35}$ C. Schaal, S. Bischoff, and L. Gaul, "Energy-based models for guided ultrasonic wave propagation in multi-wire cables," International Journal of Solids and Structure 64-65, 22-29 (2015).

${ }^{36} \mathrm{~J} . \mathrm{Xu}, \mathrm{X}$. Wu, and P. Sun, "Detecting broken-wire flaws at multiple locations in the same wire of prestressing strands using guided waves," Ultrasonics 53, 150-156 (2013).

${ }^{37}$ E. Glushkov, N. Glushkova, and S. Fomenko, "Wave energy transfer in elastic half-spaces with soft interlayers," Journal of the Acoustical Society of America 137, 1802-1812 (2015). 